\title{
R-spondins are involved in the ovarian differentiation in a teleost, medaka (Oryzias latipes)
}

\author{
Linyan Zhou ${ }^{1,3}$, Tapas Charkraborty ${ }^{2}$, Xiangguo Yu' ${ }^{1}$ Limin Wu' ${ }^{1}$ Gang Liu', Sipra Mohapatra', Deshou Wang ${ }^{1 *}$ \\ and Yoshitaka Nagahama ${ }^{4^{*}}$
}

\begin{abstract}
Background: In mammals, R-spondin (Rspo), an activator of the Wnt/ß-catenin signaling pathway, has been shown to be involved in ovarian differentiation. However, the role of the Rspo/Wnt/ $/$-catenin signaling pathway in fish gonads is still unknown.

Results: In the present study, full-length cDNAs of Rspo 1, 2 and 3 were cloned from the gonads of medaka (Oryzias latipes). The deduced amino acid sequences of mRspo 1-3 were shown to have a similar structural organization. Phylogenetic analysis showed that Rspo1, 2 and 3 were specifically clustered into three distinct clads. Tissue distribution revealed that three Rspo genes were abundantly expressed in the brain and ovary. Real-time PCR analysis around hatching (S33-5dah) demonstrated that three Rspo genes were specifically enhanced in female gonads from S38. In situ hybridization (ISH) analysis demonstrated that three Rspo genes were expressed in the germ cell in ovary, but not in testis. Fluorescence multi-color ISH showed that Rspol was expressed in both somatic cells and germ cells at 10dah. Exposure to ethinylestradiol (EE2) in XY individuals for one week dramatically enhanced the expression of three Rspo genes both at Odah and in adulthood.
\end{abstract}

Conclusions: These results suggest that the Rspo-activating signaling pathway is involved in the ovarian differentiation and maintenance in medaka.

Keywords: Rspo1, 2, 3, Molecular cloning, Expression profiles, Ovarian differentiation

\section{Background}

Sex determination in mammals occurs through inheritance of the $\mathrm{X}$ or $\mathrm{Y}$ sex chromosome from the parents. In mice, the presence of the male-determining Sry gene directs the undifferentiated gonad to develop into a testis by promoting the expression of Sox9 and Fgf9 $[1,2]$. Early ovarian development has long been thought of as a default pathway switched on passively by the absence of Sry gene. Recent genetic and transcriptomic studies challenge this view and show that two master pathways simultaneously repress male-specific genes

\footnotetext{
* Correspondence: wdeshou@swu.edu.cn; nagahama.yoshitaka.mh@ehime-u. ac.jp

'Key Laboratory of Freshwater Fish Reproduction and Development (Ministry of Education), School of Life Science, Southwest University, Chongqing 400715, P.R. China

${ }^{4}$ Institution for Collaborative Relations, Ehime University, 3 Bunkyo-cho, Matsuyama 790-8577, Japan

Full list of author information is available at the end of the article
}

and activate female-specific genetic cascades. This antagonistic action is maintained from embryonic stages to adulthood [3]. Several reports revealed that a Foxl2leading pathway and Rspo1-activating signaling pathway act independently and complementary to each other to promote ovarian development [4-6].

Studies suggest that all four members of the Rspo family play a key role in embryogenesis, development and tumorigenesis. The mammalian Rspo family is comprised of 4 members (Rspo1-4) with a similar domain organization and regulates the WNT signaling pathway via a common mechanism [7]. R-spondins function as ligands of the orphan receptors LGR4 and LGR5 to regulate Wnt/ $\beta$-catenin signaling $[8,9]$. Disruption of the human $R S P O 1$ gene in a recessive syndrome was characterized by XX sex reversal, palmoplantar hyperkeratosis and a predisposition to squamous cell carcinoma of the skin [10]. Additionally, RSPO1 was also demonstrated as

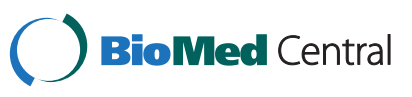


a potent and specific mitogen for the gastrointestinal epithelium, in order to promote the proliferation of intestinal crypt cells [7]. Rspo2 also appears to play an essential role in muscle development in both mouse and Xenopus embryos [11]. Since Rspo2(-/-) mice exhibited midfacial skeletal defects, lim loss and lung hypoplasia, it might be indicated that Rspo2 regulates midfacial, limb, and lung morphogenesis during development through the Wnt/ß-catenin signaling [12]. Mutation of the Rspo2 gene resulted in the formation of short hair on the head, face, and lower legs in the Portuguese water dog [13]. Knockdown of Rspo3 in Xenopus embryos induces vascular defects suggesting its key role in vasculogenesis and angiogenesis. Targeted disruption of mouse Rspo3 leads to embryonic lethality caused by vascular defects and remodeling of the vascular plexus in the placenta or impaired formation of the labyrinthine layer of the placenta [14]. Congenital mutations in RSPO4 resulted in anonychia with the absence of all fingernails and toenails in humans, and RSPO4 mutations preferentially clustered in the furin-like cysteine-rich domains $[15,16]$.

Recently, much attention has been paid to the role of the Rspo1-activating signaling pathway in the reproductive system, especially in early sex determination and differentiation. In vertebrates, Rspo1 displays a conserved, female-specific increase in expression in several species [17-19]. Investigations in mammalian species have demonstrated that RSPO1 is postulated to switch on ovarian determination and differentiation by synergizing with specific Wnt ligands to stabilize the intracellular canonical $\beta$-catenin signaling pathway, which in turn activates ovarian differentiating genes in the bipotential gonad [20]. Mutations of RSPO1 in humans induce testis formation and male development in $\mathrm{XX}$ individuals, in the absence of $S R Y$ [10]. In the mouse, ovarian differentiation requires activation of the RSPO1/WNT/ $\beta$-catenin signaling pathway in both somatic cells and germ cells. In Rspo1 ${ }^{-/-} \mathrm{XX}$ gonads, severe impairments i.e. germ cell proliferation, expression of the early meiotic marker Stra 8 and entry into meiosis were observed. The author proposed that RSPO1/ $\beta$-catenin signaling is involved in meiosis in fetal germ cells and contributes to the cellular decision of germ cells to differentiate into oocytes or sperms [21]. In the goat gonads, both Rspo1 and 2 showed a female-specific expressional profile from 36 day post coitus $(d p c)$ to adulthood. Therefore, goat Rspo1 was correlated with germ line cell differentiation before and during meiosis, while Rspo 2 was considered as a candidate gene for ovarian differentiation. Goat Rspo4 was also specifically expressed in both the XX female gonad from 50 to $90 d p c$, although only very faintly. However, Rspo3 was equally expressed in XX and XY gonads [5]. Except for goat, the expression and potential roles of all Rspo family members in other vertebrates are largely unknown.

Medaka has been used as an ideal model to study sex determination and differentiation with XX-XY genetic system and small genome [22]. DMY/Dmrt1b has been identified as the male sex determining gene of medaka, which initiates the development of testes in $\mathrm{XY}$ males by inhibiting male primordial germ cell (PGC) proliferation at the sex-determining stage $[23,24]$. Conversely, it is well accepted that estrogen is essential for ovarian differentiation and maintenance in female fish [25-27]. It was well documented that estrogen is necessary for the maintenance of Rspo1 expression in a direct or indirect manner in oviparous species [19]. Recent studies revealed that Wnt signaling is implicated in multiple processes of male and female gonadal development in rainbow trout [28]. Therefore, it is essential and critical to explore whether Rspo/Wnt/ $\beta$-catenin signaling pathway plays a key role in fish sex determination and differentiation, just like its role in mammals. In this study, we investigated the temporal and spatial expression profiles of Rspo1, 2 and 3 in the gonads during early ontogenic stages, meanwhile their expression profiles by steroid treatment were also examined. To our knowledge, this is the first report that Rspo family members might be critical for ovarian differentiation and maintenance in fish.

\section{Results}

\section{Sequence analysis}

In this study, three Rspo genes were cloned in medaka. The ORF of Rspo1, 2 and 3 contained 810 bp, 738 bp and 984 bp encoding 270, 246 and 328 amino acids (a), respectively. Sequence analysis revealed that medaka Rspo1 displayed higher identity to tilapia (84\%) and zebrafish (74\%) than its mammalian counterpart i.e. human (62\%), mouse (62\%), chicken (64\%). The putative amino acid sequence of medaka Rspo2 also revealed higher similarity to zebrafish (73\%) than human (69\%), mouse (68\%), chicken (69\%) and Хenopus (69\%). However, the deduced amino acid sequence of medaka Rspo3 showed relative lower homology to zebrafish (58\%), human (46\%), mouse (46\%), chicken (45\%), and Xenopus (41\%) Rspo3.

Similar to mammalian species, medaka Rspol and 2 contain five exons by a structural analysis. However, fish Rspo3 from medaka and zebrafish includes 6 exons. Three fish Rspo family proteins share substantial structural homology and possess one signal peptide at the $\mathrm{N}$-terminal, two or three conserved cystine-rich furinlike domains (FU) and a thromobospondin-1 domain (TSP-1) (Figure 1). The C-terminal sequences of the three Rspo proteins were found to be less conservative. 


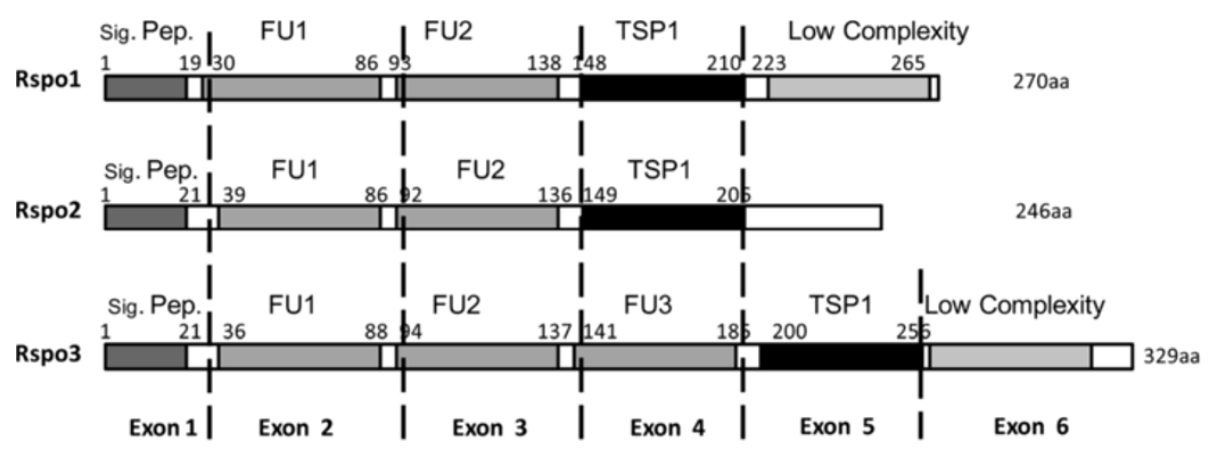

Figure 1 Exon organization and conserved domain structure of three medaka R-spondins. The medaka Rspo 1 and 2 protein genes share a common organization, each consisting of five coding exons corresponding to predicted structural domains. Predicted domains include a leading signal peptide (Sig. Pep), two furin-like type Cys-rich domains (FU1, FU2), a thrombospondin-type domain (TSP) and low complexity (white color). Additionally, Rspo3 protein possesses an extra furin-like type Cys-rich domain (FU3) located in exon 4. Conserved domain residues and exon boundaries are indicated by rectangle (gray or black) and dotted lines, respectively.

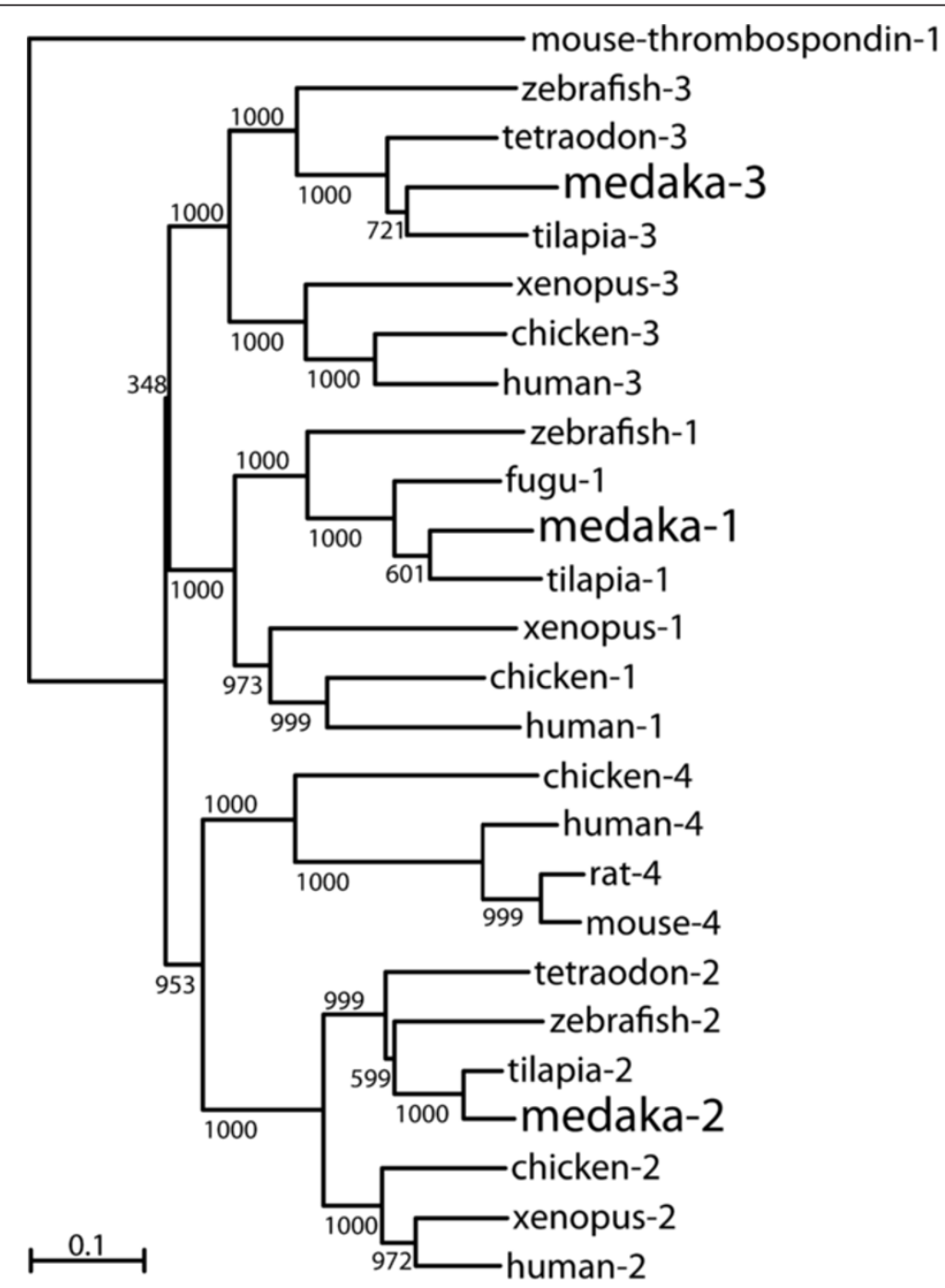

Figure 2 Phylogenetic tree of Rspo family proteins of vertebrates was constructed by using mouse thrombospondin-1 as an outgroup. Values on the tree represent bootstrap scores of 1000 trials, indicating the credibility of each branch. Branch lengths are proportional to the number of amino acid changes on the branch. Refer to Materials and Methods for GenBank accession nos. 


\section{Phylogenetic analysis}

To understand the phylogenetic relationship of RSPO family members among vertebrates, a phylogenetic tree was constructed based on the amino acid sequences of Rspo1, 2, 3 and 4 from different species (Figure 2). Phylogenetic analysis demonstrated that Rspo1, 2 and 3 from medaka along with their mammalian counterparts were clustered into three distinct clads. However, Rspo4 couldn't be isolated from the available genome DNA databases in fish.

\section{Tissue distribution}

Various tissues were collected from adult medaka for RNA extraction and cDNA synthesis which was used as templates for real time PCR analysis. The tissue distribution analysis revealed that Rspol and 2 were ubiquitously expressed in the brain, liver, heart, intestine, kidney, ovary and testis, with dominant expression in the brain, liver and ovary. Rspo3 was expressed at almost the same level in all the checked tissues except testis. The mRNA levels of Rspo1 and 2 were much higher than that of Rspo3 in gonads. Importantly, sexually dimorphic expression profiles of these genes were found in the gonads with much higher levels in the ovary than in the testis (Figure 3).

\section{Ontogenic expression of Rspo1, 2 and 3 by real-time PCR}

In medaka, the first morphological sex difference manifested in the gonads reflects that the female-specific
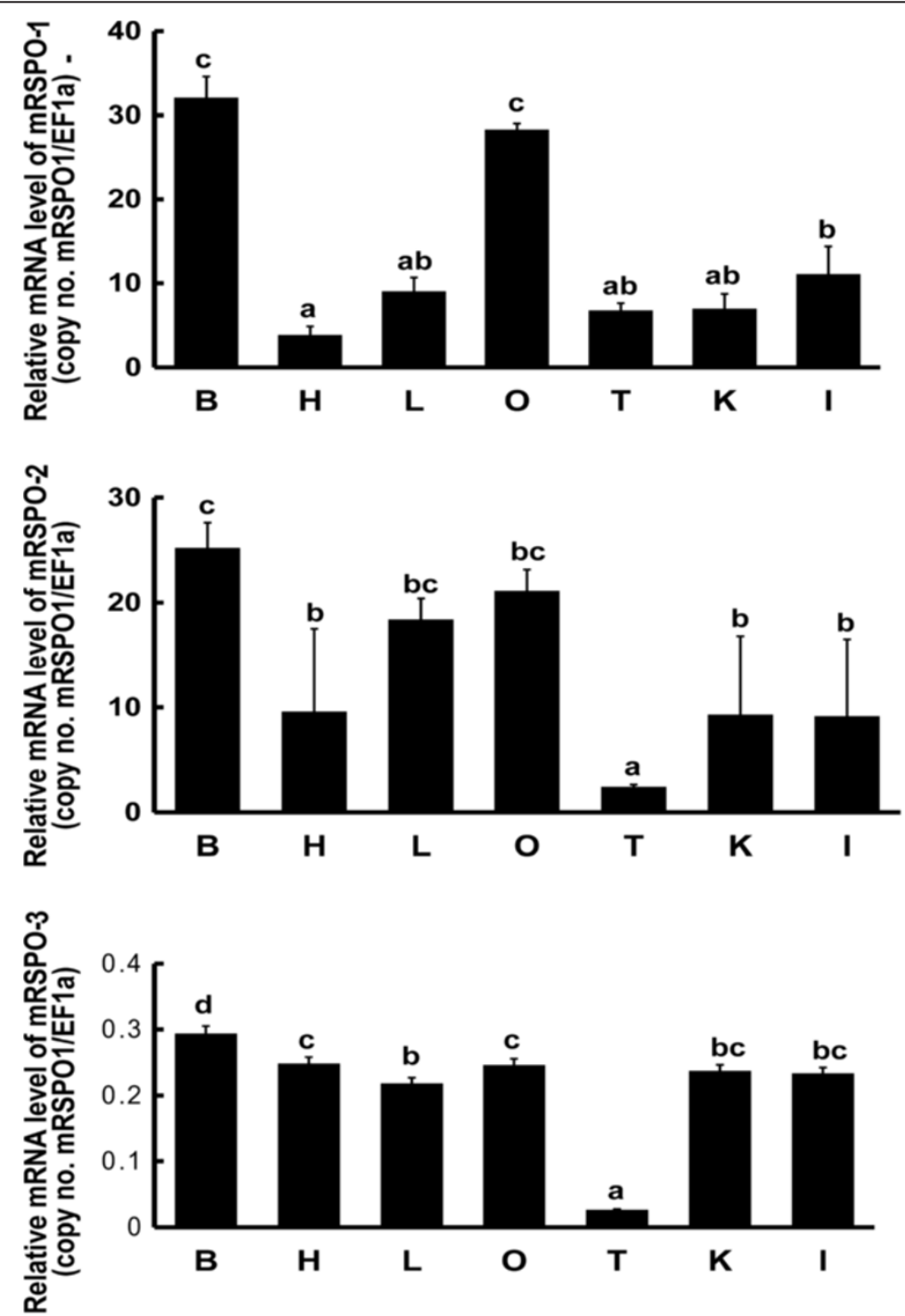

Figure 3 Real-time PCR analysis of Rspo 1, 2 and 3 in various tissues of adult medaka. B, brain; H, heart; L, liver; O, ovary; T, testis; K, kidney; I, intestine. Relative mRNA level (copy number of each gene/EF1-a) represents the mean \pm S.E. of samples from at least 3 different fish. Values with different letters indicate significant difference $(P<0.05)$ of each gene in different tissues. 
germ cell proliferation starts from stage 38 before hatching [29]. A real-time PCR analysis was carried out to investigate the expression profiles of three Rspo genes during the critical period of sex determination/differentiation (Figure 4). Intriguingly, female-specific increase in Rspo1, 2 and 3 expression profiles were detected at S38 and S39 when the first morphological sexual differentiation occurs in medaka, while it was decreased from Odah. In contrast, in males, the expression of all three Rspo genes remained at a much lower level during those stages.

\section{Expression of Rspo1, 2 and 3 in the gonads by ISH}

ISH analysis revealed that three Rspo genes were abundant in the ovary, but barely detectable in the testis. Single-color ISH analysis showed that both Rspo1 and 2 were predominantly expressed in the germ cells and germ cell surrounding cells at S38 and 0dah (Figure 5). Later on, their expressions were restricted to the cytoplasm of oogonia, oocytes, primary oocytes and corticalalveolar stage oocytes from 30dah to adulthood. However, they were not found in late cortical-alveolar stage oocytes, vitellogenic stage oocytes, or the follicular cell layer. The expression of Rspo3 in XX female gonads could be detected in the adult stage (Figure 5), but was barely detectable during the early ontogenic stages by ISH (data not shown).

Fluorescence multi-color ISH analysis demonstrated that Rspo1 was expressed both in the germ cell (overlapping with germ cell marker gene, Vasa) and somatic cell surrounding the germ cell (overlapping with follicular cell marker gene, Foxl2) at 10dah (Figure 6). The expression of fish Rspo genes was not detected in the somatic cell by traditional ISH method, which

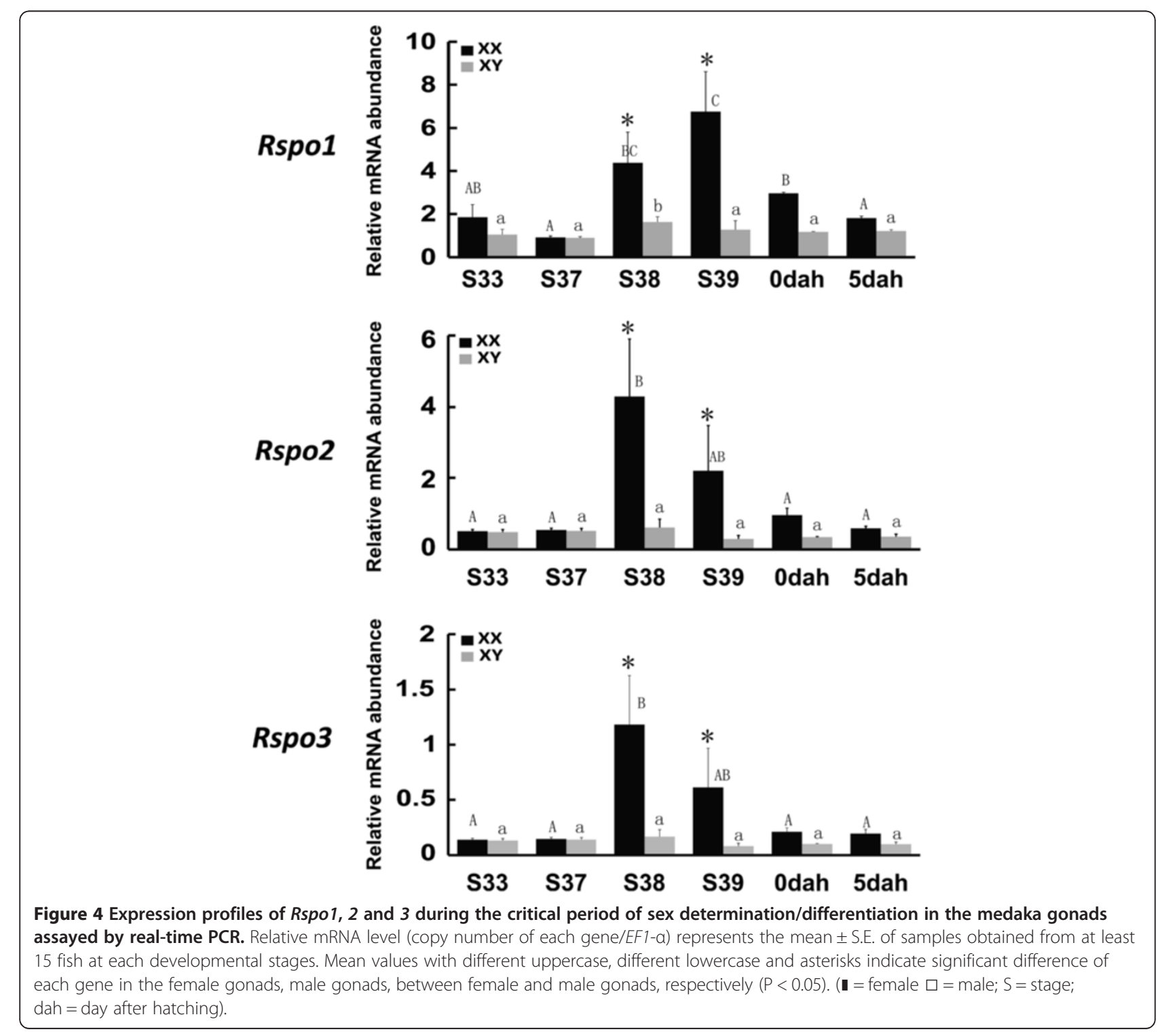




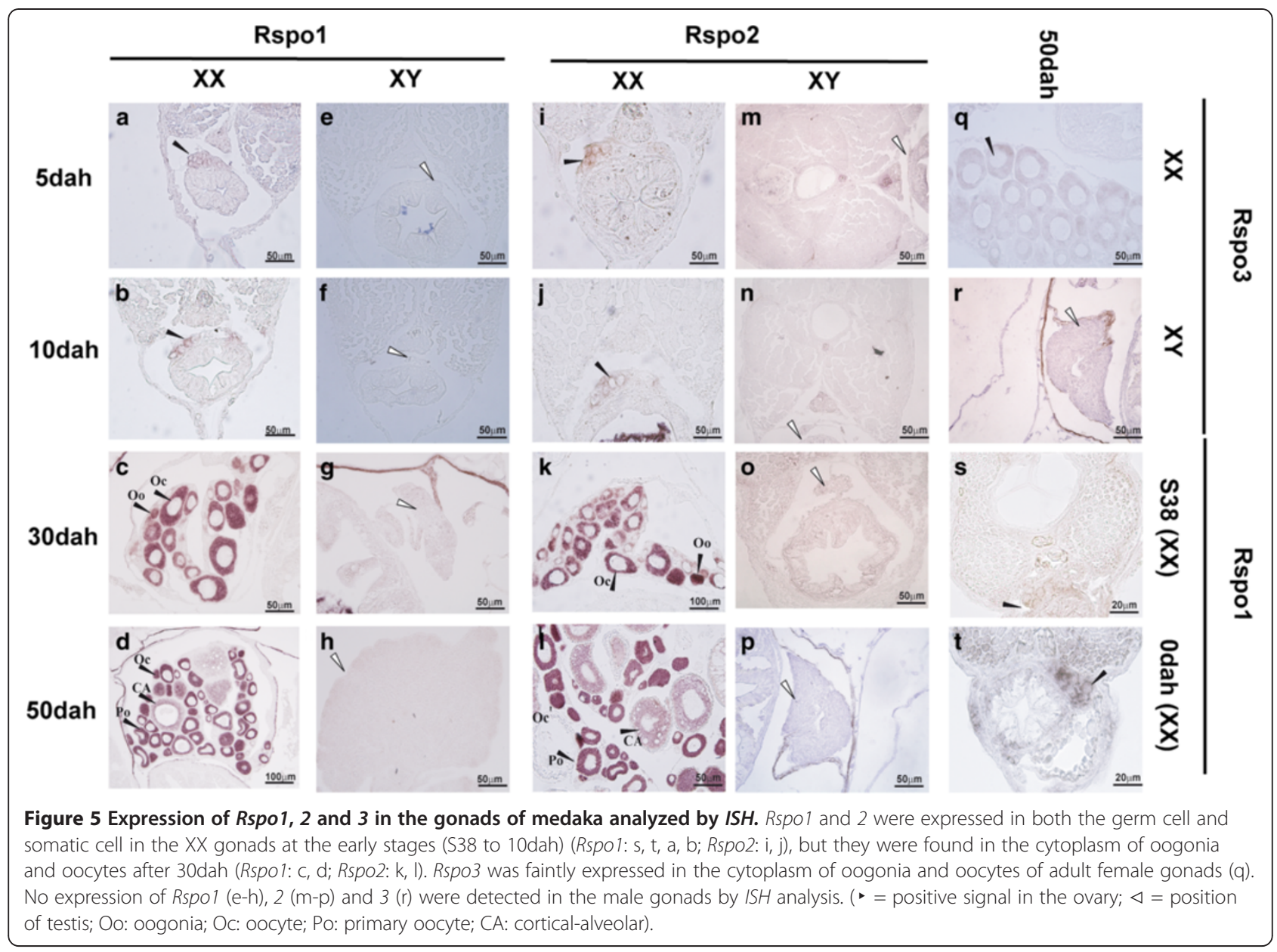

might due to their low expression in the somatic cell surrounding the germ cells.

\section{Effect of steroid treatment on the expression of Rspo1, 2 and 3}

At Odah, treatment with EE2 significantly increased the expression of Rspo1, 2 and 3 in XY embryos, but levels were much lower than their expression level in control $\mathrm{XX}$ embryos (Figure 7a). Moreover, treatment of adult $\mathrm{XY}$ fish with EE2 caused significant enhancement of Rspo1, 2 and 3 expressions comparing with normal XX female levels (Figure 7b). ISH revealed that Rspo1, 2 and 3 were up-regulated in the EE2 treated XY gonad at S37, Odah and adult stage (Additional file 1: Figure S1).

\section{Discussion}

Three members of the Rspo family were cloned and characterized from a teleost fish, medaka. Interestingly, medaka Rspo1, 2 and 3 showed a sexually dimorphic expression profile with female-specific up-regulation during the critical period of sex determination and differentiation and later developmental stage. Thus, the abundant expression of these three Rspo genes in the female gonad indicated that Rspo-activating pathway might be required for ovarian differentiation and maintenance in fish.

In vertebrates, Rspo1 displayed a female-specific increase in the gonads of humans, mice, goats, chickens and reptiles during the critical period of sex determination/differentiation [5,17-19]. To date, the expression of Rspo1, 2 and 3 had merely been identified in goats. It has been reported that goat Rspo 2 was expressed with a female-specific profile from the crucial stage of sex determination until adulthood. However, goat Rspo3 was expressed equally in females and males [5]. In medaka, it has been shown that sex determination occurred around stage 38 before hatching [29]. At this stage, other than the germ cell markers in females, as well as $D M Y$ and GSDF in males, all known genes have been shown to be expressed without any sexual dimorphism at this stage [29-31]. Our real-time PCR results demonstrated that medaka Rspo1, 2 and 3 expression was specifically upregulated from S38 along with the expression of a germ cell marker (Vasa) and meiosis marker (Spo11) in the female gonads. A previous report demonstrated that $\mathrm{RSPO} 1 / \beta$-catenin signaling is involved in meiosis in fetal 

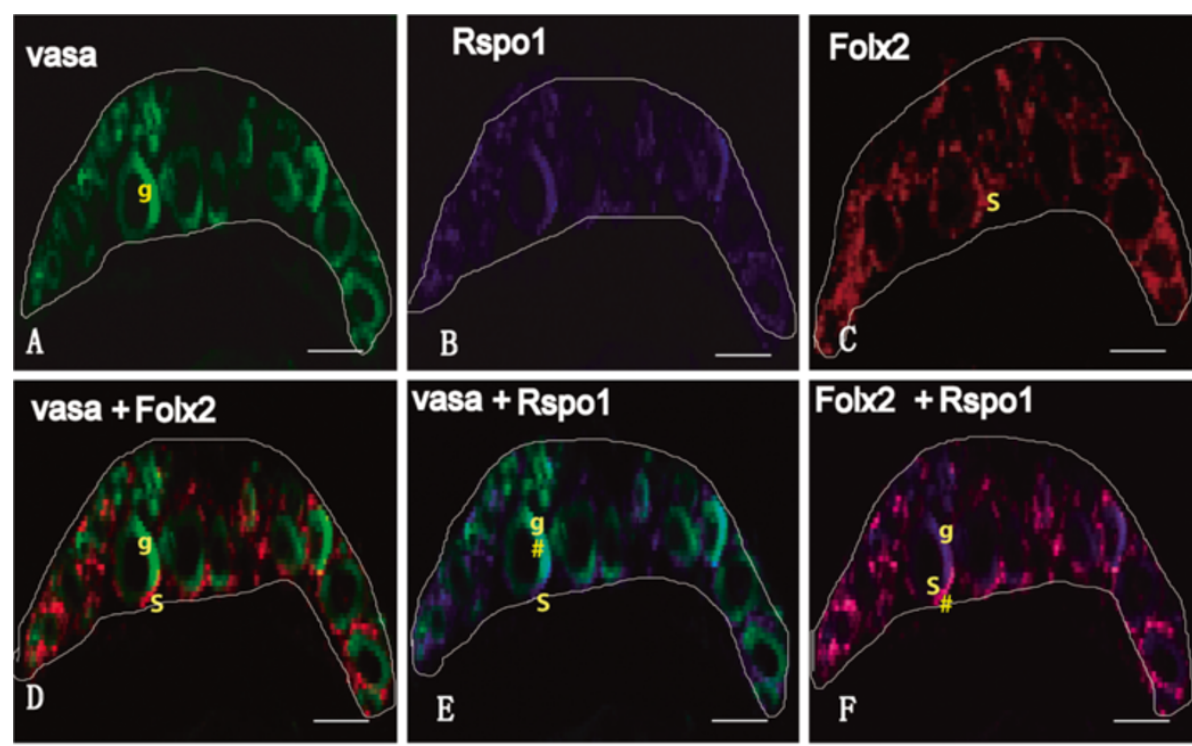

Figure 6 Localization of Vasa, Rspo1 and FoxI2 in 10dah XX gonads. RNA probes were labeled with FITC, DIG or Biotin, respectively. Stained sections were observed under a confocal laser microscope. Photographs in A-F show the same area of the XX gonad. The upper panels were the signals of Vasa (green) in the germ cells (A), Rspol (blue) (B) and Fox/2 (red) in the somatic cells (C). The lower panels were the signals of two genes by overlapping. D: Vasa and Foxl2; E: Vasa and Rspo1; F: Rspol and FoxI2. The \# in E indicates co-localization of Vasa and Rspol in the germ cells. The \# in F indicates co-localization of Fox 2 and Rspo1 in the somatic cells. $g$ indicates germ cell and $\mathrm{S}$ indicates somatic cell. The boundary of gonad is marked by white color lines.

germ cells and contributes to the cellular decision of germ cells to differentiate into oocytes or sperms [21]. The expression profiles of medaka Rspo1, 2 and 3 during the early sex determination/differentiation stages imply that Rspo signaling might be also required to initiate meiosis in the germ cells of medaka. Therefore, the female-specific expression of the three Rspo genes in medaka suggests that they lie upstream in the genetic cascade of female sex determination and differentiation in teleosts.

The sub-cellular distribution of Rspo1 protein during the critical period of sex determination has been well investigated in mice, goats and chickens. In the E14.5 female mouse gonad, the protein was mainly located in germ cells and somatic cells. In the E8.5 chicken ovary, it was widely expressed in the cytoplasm and on the cell surface in the outer cortical zone including both germ and somatic cells, just prior to the onset of meiosis [19]. In goat, the protein was detected around both somatic and germinal cells in the cortical area as early as 36$40 d p c$. At $50 d p c$, the strongest Rspo1-specific signal was found around the germ cells in goats [5]. Our multicolor ISH data revealed that medaka Rspo1 mRNA was expressed in both somatic cells (overlapping with Foxl2)
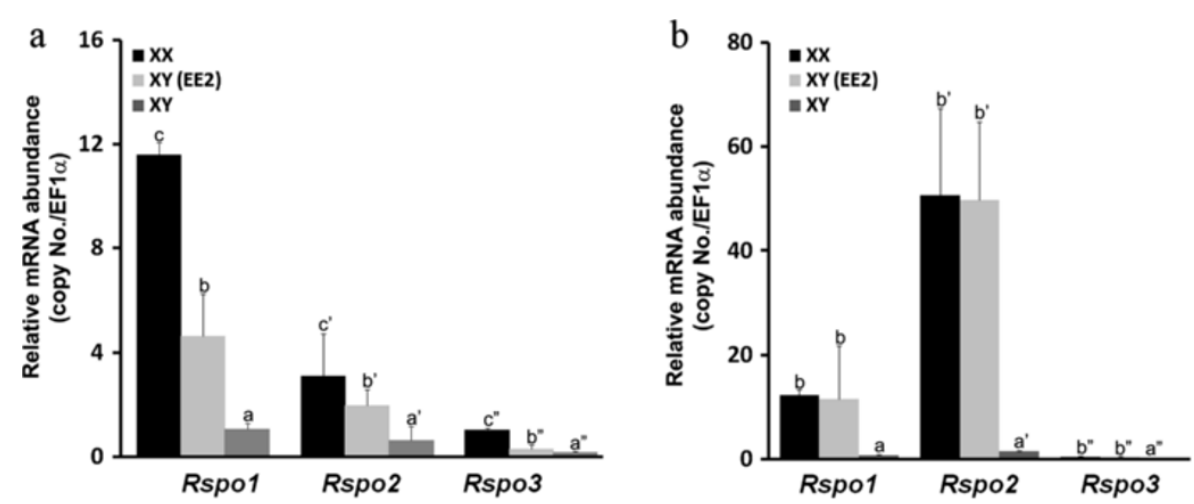

Figure 7 (a) Changes of the expression profiles of Rspo1, 2 and 3 after EE2 treatment at 0dah ( $\mathbf{n}=15)$. (b) Effect of EE2 treatment on Rspo 1, 2 and 3 expression in adult $X Y$ fish $(n=6)$. Values (mean \pm S.E.) represent the relatively mRNA abundance of Rspo 1, 2 and 3 . Values with different letters indicate significant difference $(P<0.05)$ of each gene. $(X X$, control; $X Y$-EE2, treated group; $X Y$, control). 
and germ cells (overlapping with Vasa) in the early stages of sex determination (10dah). However, strong expression of Rspo1 was only detected in the cytoplasm of oocytes at a much later stage. Additionally, up to now, no reports are available on the cellular distribution of Rspo 2 and 3 in vertebrates. In this study, both Rspo 2 and Rspo3 showed the same expression profiles as Rspo1 in the gonads during different ontogenic stages in the ISH analysis. Concisely, the female-specific expression profile in the somatic (pre-follicular) cells and germ cells during early sexual differentiation suggests a possible role of fish Rspo proteins in both folliculogenesis and development of germ cells.

The role of germ cells in sex determination and differentiation differs between mammals and fish. In mammalian species, the presence of PGC doesn't play an important role in somatic sexual differentiation. Unidirectional signaling from the soma to germ cells has been found to be important for sex determination and differentiation [32]. In fish, previous reports showed that gonadal somatic cells are predisposed to male development in a cell-autonomous fashion. Therefore, in medaka, germ-cell-deficient adults displayed a female-to-male secondary sex reversal phenotype [33]. Similarly, the ablation of germ cells resulted in the generation of sterile males, indicating that the germ cell line is essential for the development of female zebrafish [34]. Therefore, germ cells are necessary for sexual dimorphism and sex differentiation in fish. In this study, the presence of Rspo protein in both somatic and germ cells in the developing ovary indicated its essential role in fish sexuality.

Estrogens play a pivotal role in ovarian differentiation and maintenance in non-eutherian vertebrates. Recent reports revealed that Foxl2 plays a decisive role in ovarian differentiation by regulating aromatase expression and possibly the entire estrogen pathway in teleosts [26]. Foxl 2 and RSPO1 double knockout resulted in sex reversal in XX mice [35]. In chicken, treatment with an aromatase inhibitor (Fadrozole) reduced the expression of Rspo1. In the present study, the expression profiles of Rspo1-3 in gonads were remarkably enhanced during a short period of exposure to estrogen, in both Odah and adult XY medaka. This result further strengthens the idea that estrogen could induce the activation of Rspo signaling pathway which is required for ovarian development in XY sex reversal females treated with EE2. Evidence in mice and humans suggests that the canonical Wnt signaling pathway promotes ovarian fate and blocks testis development. Duplication of the distal portion of chromosome 1p, which includes both WNT4 and RSPO1, overrides the male program and causes male-tofemale sex reversal in XY patients. Ectopical expression of $\beta$-catenin in the somatic cells of $\mathrm{XY}$ gonads disrupts the male program and results in male-to-female sex- reversal [36,37]. Our preliminary studies showed that over-expression of Rspo1 in XY medaka disrupts male development, and induces ovarian development (data not shown). Therefore, we hypothesized that like in mammals, the proper development of ovaries requires the interaction and complement of Rspo-activating signaling pathway and Foxl2-leading estrogen producing pathway. However, further investigation is required to test this presumption.

\section{Conclusions}

These results suggest that the Rspo-activating signaling pathway is involved in the ovarian differentiation and maintenance in medaka. Our data also support that estrogen producing pathway and Rspo-activating signaling pathway might be complementary in female sex determination/differentiation in fish.

\section{Methods}

\section{Fish strains and husbandry}

The QurtE strain of medaka (Oryzias latipes) was used for gene cloning and expression analysis. All fish were maintained under a 14-h light, 10-h dark photoperiod prior to use. Both $\mathrm{Y}$ chromosome-derived lucorphore and genomic PCR (DMY-F, DMY-R) were used to identify the genetic sex of fish before experiments. All in vivo experiments and fish maintenance were conducted following protocols and procedures approved by Institutional Animal care and use committee at the National Institute for Basic Biology, Japan.

\section{Molecular cloning}

A $540 \mathrm{bp}$ fragment encoding Rspo1 was isolated from the medaka ovary by gene specific primers (1-F1, 1-R1) designing according to the EST sequences of medaka (K05119-53_A06). Subsequently four gene-specific primers were designed to amplify the 5'- and 3'-end cDNA sequence by SMART 5 -rapid amplication of cDNA ends (RACE) and 3'-RACE (1-F2, 1-F3, 1-R2, 1-R3) according to the manufacturer's instructions. The open reading frame (ORF) of Rspo2 was obtained from the medaka ovary by gene specific primers (2-F, 2-R) based on the available database (ENSORLT00000025646). A partial sequence of Rspo3 was amplified (3-F1, 3-R1) in the medaka ovary basing on the available sequence from medaka genome databases (ENSORLT00000007234). The full ORF of Rspo3 was obtained by RACE (3-F2, 3-R2). All PCR products were ligated into the pGEM-T easy vector (Promega, Madison, WI) and sequenced using an ABI Prism 3100 sequencer (Applied Biosciences, Branchburg, NJ). 


\section{Phylogenetic analysis}

The deduced amino acid sequences of medaka Rspo1, 2 and 3 and their counterparts in other vertebrates, as well as Rspo4 from mammalian species were aligned using Clustal W. A phylogenetic tree was generated with PHYLIP software by the neighbor-joining method [38] using mousethromobosponding 1 (NP_035710) as an out-group. Values on the tree represent the bootstrap scores of 1000 trials, indicating the credibility of each branch. The GenBank accession nos. of sequences used in this study are as follows, human-RSPO1(NP_001033722), chicken-Rspo1 (XP_4177 60), Xenopus-Rspo1 (NP_001121500.1), zebrafish-Rspo1 (NP_001002352), tilapia-Rspo1 (JF276456), medaka-Rspo1 (JF263584), fugu-Rspo1 (CAAB02002595), human-RSPO2 (XP_001134914), chicken-Rspo2 (XP_418383), ХenopusRspo2 (NP_001088999), zebrafish-Rspo2 (XP_001919458), medaka-Rspo2 (JF263585), tilapia2 (XP_003453515.1), human-RSPO3 (NP_116173), Xenopus-Rspo3 (AAV31038), zebrafish-Rspo3 (NP_001017358), chicken-Rspo3 (XP_419 752), tetraodon-Rspo3 (CAG12893), medaka-Rspo3 (JF26 3586), tilapia3 (XP_003443788.1), human-RSPO4 (EAX1 0652), mouse-Rspo4 (EDL05937), rat-Rspo4 (XP_575261), chicken-Rspo4 (BAL43044).

\section{Tissue distribution}

For the tissue distribution analysis, total RNA was extracted from brain, heart, liver, ovary, testis, kidney and intestine of adult medaka, according to the manufacturer's instructions (RNeasy Mini kit, QIAGEN) with RNase-free DNase treatment. Subsequently, reverse transcription for cDNA was conducted (Omniscript RT kit, QIAGEN), and quantitative RT-PCR was carried out to check the levels of Rspo1, 2 and 3 in various tissues. The data were analyzed using one-way ANOVA and the least significant difference on the GraphPad Prism 5 software (San Diego, CA, USA).

\section{Preparation of samples for ISH}

Whole body specimens of both XX and XY medaka fry at different developmental stages were fixed in $4 \%$ paraformaldehyde (Nacalai tesque, Kyoto, Japan) in 0.85x PBS at $4{ }^{\circ} \mathrm{C}$ as described previously [39]. Probes of sense and antisense digoxigenin (DIG) labeled RNA strands were transcribed in vitro with a RNA labeling kit (Roche Diagnostics GmbH, Mannheim, Germany) from plasmid DNA containing the ORF of Rspo1, 2 and 3.

To detect the cellular localization of Rspo1 during early embryogenesis, fluorescence multi-color ISH of Rspo1, Vasa and Foxl2 was performed as described previously [40]. Briefly, probes were labeled with fluorescein isothiocyanate (FITC), or DIG or Biotin (Roche, Germany). Horseradish peroxidase-conjugated anti-FITC, anti-DIG and anti-biotin antibodies were used for the detection, respectively. For detection of the signals, a TSA Plus
Fluorescein/TMR system was used (Inc., Waltham, MA). Signals were observed and photographed by confocal microscope (Zeiss 710, Carl-Zeiss Germany).

\section{Real-time PCR}

For ontogenic expression analysis of three Rspo genes in the medaka gonads, triplicates of five beheaded embryos were collected from both female and male at stage (S) 33, S37, S38, S39, 0dah and 5dah. Subsequent total RNA extraction, cDNA synthesis and real-time PCR were carried out to check the expression of Rspo1 (1-F4, 1-R4), 2 (2-F2, 2-R2) and 3 (3-F4, 3-R2) as described previously [41]. Data were expressed as the mean \pm SE for the 3 replicates. A Kruskal-Wallis test was used to determine significant difference $(\mathrm{P}<0.05)$ with GraphPad Prism 5 software (GraphPad Software, San Diego, CA).

\section{Treatment with steroid}

Vast investigations have proved that exposure to estrogenic chemicals, including natural and synthetic estrogens caused feminization responses or complete sex reversal in male fish. A synthetic estrogen, ethinylestradiol (EE2) is an effective estrogenic chemicals could

Table 1 Primer sequences used in molecular cloning and real-time PCR analysis

\begin{tabular}{lll}
\hline Primer & Sequence & Purpose \\
\hline Rspo1-F1 & TGGGACTGGTGGCGCTGGCGATG & $\begin{array}{l}\text { fragment } \\
\text { amplification }\end{array}$ \\
Rspo1-R1 & GCCTTCTTAAACCCACATGT & \\
Rspo2-F1 & ATGCAGTTCGACTCTTCTC & \\
Rspo2-R1 & TCACTGGCTGGAGCGAGCAG & \\
Rspo3-F1 & GCCATGCAATTACAAGTCATCTC & \\
Rspo3-R1 & CTACTGCACAAGGCTGTGCTCGGGG & \\
Rspo1-F2 & CCCAGAAGGGAATAACCGATGCACAC & 3'- RACE \\
Rspo1-F3 & CACACAGTGGGCGATGCTACGTCAGC & \\
Rspo3-F2 & GGCAGACCCTGCCCTTGACCACAG & \\
Rspo3-F3 & GGCAGACCCTGCCCTTTGACCACAG & \\
Rspo1-R2 & CGCTCCAAGAAGATAAAGAGCTTGGG & 5'- RACE \\
Rspo1-R2 & CTCGTTCTGCCTTCTCGCCTCCC & \\
Rspo1-F4 & AAGTGCGTTGGCCCAAAACACCG & real-time PCR \\
Rspo1-R4 & TCCCATCTITCCCTCTCGCCCTAGTC & \\
Rspo2-F2 & ACCACCCAAGGACACAATCC & \\
Rspo2-R2 & GTGCTTCCCTGAACCACCTC & \\
Rspo3-F4 & AAGAGGATCGGGATGAAGCA & \\
Rspo3-R2 & TCACACTCCGACCTGCACTT & \\
EF1-a-F & CAGCTTCAACGCTCAGGTCAT & \\
EF1-a-R & TGAACTTGCAGGCGATGTGA & \\
DMY-F & CCGGGTGCCCAA GTGCTCCCGCTG & \\
DMY-R & GATCGTCCCTCCACAGAGAAGAGA & \\
\hline & & \\
Renetic sex
\end{tabular}


cause the feminization or sex reversal in vertebrates including fish $[42,43]$.

The effect of EE2 on the expression of the three Rspo genes was evaluated by short term immersion of either fertilized eggs or $\mathrm{XY}$ adults in aerated fresh water with or without EE2 $(10 \mathrm{ng} / \mathrm{L})$ dissolved in ethanol (Wako Pure Chemicals, Japan). The fertilized eggs were treated by EE2 until hatching as described previously and $\mathrm{XY}$ embryos carrying lucorphore were sampled at $8 \mathrm{dpf}$ (0dah) [44]. RNA extraction and cDNA synthesis were obtained from 3 samples and 5 embryos without head for each sample. For adult XY fish, six male medaka were randomly selected and maintained in aerated fresh water with EE2 $(10 \mathrm{ng} / \mathrm{L})$ for one week. The gonads of EE2 treated adult fish were dissected separately for RNA extraction and subsequent cDNA synthesis. Simultaneously, fertilized eggs or adult XY male fishes were assigned to immersion in the same amount of vehicle ethanol in both experiments as control groups, and then RNA extraction and cDNA synthesis also were prepared according to the methods in EE2 treated group. Finally, real-time PCR was carried out to investigate the expression profiles of Rspo1, 2 and 3 according to the methods aforementioned. Results are presented as the mean $\pm \mathrm{S}$. E. of data from triplicates. The data were analyzed using one-way ANOVA and the least significant difference on the GraphPad Prism 5 software (San Diego, CA, USA). Additionally, ISH analysis were carried out to further check the expression changes of Rspo1, 2 and 3 genes in EE2 treated XY gonads at S37, Odah and adult stage. In this experiment, EE2 treatment for $\mathrm{XY}$ individual at each stage followed the aforementioned protocol.

Primer sequences used for RT-PCR, RACE and realtime PCR are listed in Table 1.

\section{Additional file}

Additional file 1: Figure S1. Expression of Rspo 1 (a-c), 2 (d-f) and 3 (g-i) in the EE2 treated XY gonads at S37, Odah and adult stage. The expressions of three genes were greatly up-regulated in XY gonad by EE2 treatment during three stages. The gonadal boundary is marked by black lines. Scale bar, $50 \mu \mathrm{m}$.

\section{Competing interests}

The authors declare that there is no financial or other potential conflict of interests.

\section{Authors' contributions \\ All authors participated in the design of this study. LZ cloned and characterized three types of Rspo genes. TC performed the multiple color ISH. XY and GL carried out the steroid treatment and tissue collection. LW performed the real-time PCR analysis and all the authors analyzed the results. SM, DW and YN drafted the manuscript. All authors read and approved the final manuscript.}

\section{Acknowledgements}

This work was supported in part by Grants-in-Aid for Scientific Research from the JSPS Postdoctoral Fellowship for Foreign Researchers, the SORST
Research Project of the Japan Science and Technology Agency, and the Ministry of Education, Science, Sports, and Culture of Japan. This work was also supported in part by Research Foundation of Talent Introduction of Southwest University, China (SWU111003), the Specialized Research Fund for the Doctoral Program of Higher Education of China, Ministry of Education (20110182120003) and grants from the National Natural Science Foundation of China (31030063 and 31201986). The authors are thankful to National Bioresource Project (NBRP)-medaka for providing the medaka strains.

\section{Author details}

${ }^{1}$ Key Laboratory of Freshwater Fish Reproduction and Development (Ministry of Education), School of Life Science, Southwest University, Chongqing 400715, P.R. China. ²Division of Molecular Environmental Endocrinology, National Institute for Basic Biology, Okazaki 444-8585, Japan. ${ }^{3}$ Japan Society for the Promotion of Science (JSPS), 8 Ichiban-cho, Chiyoda-ku, Tokyo 102-8472, Japan. ${ }^{4}$ Institution for Collaborative Relations, Ehime University, 3 Bunkyo-cho, Matsuyama 790-8577, Japan.

Received: 21 June 2012 Accepted: 13 November 2012

Published: 7 December 2012

\section{References}

1. Sinclair AH, Berta P, Palmer MS, Hawkins JR, Griffiths BL, Smith MJ, Foster JW, Frischauf AM, Lovell-Badge R, Goodfellow PN: A gene from the human sex-determining region encodes a protein with homology to a conserved DNA-binding motif. Nature 1990, 346(6281):240-244.

2. Koopman P, Gubbay J, Vivian N, Goodfellow P, Lovell-Badge R: Male development of chromosomally female mice transgenic for Sry. Nature 1991, 351(6322):117-121.

3. Angelopoulou R, Lavranos G, Manolakou P: Sex determination strategies in 2012: towards a common regulatory model? Reprod Biol Endocrinol 2012, 22(10):13.

4. Garcia-Ortiz JE, Pelosi E, Omari S, Nedorezov T, Piao Y, Karmazin J, Uda M, Cao A, Cole SW, Forabosco A, et al: Foxl2 functions in sex determination and histogenesis throughout mouse ovary development. BMC Dev Biol 2009, 9:36.

5. Kocer A, Pinheiro I, Pannetier M, Renault L, Parma P, Radi O, Kim KA, Camerino G, Pailhoux E: R-spondin1 and FOXL2 act into two distinct cellular types during goat ovarian differentiation. BMC Dev Biol 2008, 8:36.

6. Schlessinger D, Garcia-Ortiz JE, Forabosco A, Uda M, Crisponi L, Pelosi E: Determination and stability of gonadal sex. J Androl 2010, 31(1):16-25

7. Kim KA, Wagle M, Tran K, Zhan X, Dixon MA, Liu S, Gros D, Korver W, Yonkovich S, Tomasevic N, et al: R-Spondin family members regulate the Wnt pathway by a common mechanism. Mol Biol Cell 2008, 19(6):2588-2596.

8. de Lau W, Barker N, Low TY, Koo BK, Li VS, Teunissen H, Kujala P, Haegebarth A, Peters PJ, van de Wetering M, et al: Lgr5 homologues associate with Wnt receptors and mediate R-spondin signalling. Nature 2011, 476(7360):293-297.

9. Carmon KS, Gong X, Lin Q, Thomas A, Liu Q: R-spondins function as ligands of the orphan receptors LGR4 and LGR5 to regulate Wnt/betacatenin signaling. Proc Natl Acad Sci USA 2011, 108(28):11452-11457.

10. Parma P, Radi O, Vidal V, Chaboissier MC, Dellambra E, Valentini S, Guerra L, Schedl A, Camerino G: R-spondin1 is essential in sex determination, skin differentiation and malignancy. Nat Genet 2006, 38(11):1304-1309.

11. Yamada W, Nagao K, Horikoshi K, Fujikura A, Ikeda E, Inagaki Y, Kakitani M, Tomizuka K, Miyazaki H, Suda T, et al: Craniofacial malformation in R-spondin2 knockout mice. Biochem Biophys Res Commun 2009, 381(3):453-458.

12. Parker HG, Chase K, Cadieu E, Lark KG, Ostrander EA: An insertion in the RSPO2 gene correlates with improper coat in the Portuguese water dog. J Hered 2010, 101(5):612-617.

13. Kazanskaya O, Glinka A, del Barco Barrantes I, Stannek P, Niehrs C, Wu W: R-Spondin2 is a secreted activator of Wnt/beta-catenin signaling and is required for Xenopus myogenesis. Dev Cell 2004, 7(4):525-534.

14. Kazanskaya O, Ohkawara B, Heroult M, Wu W, Maltry N, Augustin HG, Niehrs $\mathrm{C}$ : The Wnt signaling regulator $\mathrm{R}$-spondin 3 promotes angioblast and vascular development. Development 2008, 135(22):3655-3664.

15. Bergmann $C$, Senderek J, Anhuf $D$, Thiel $C$, Ekici AB, Poblete-Gutierrez $P$, van Steensel M, Seelow D, Nurnberg G, Schild HH, et al: Mutations in the gene encoding the Wnt-signaling component R-spondin 4 (RSPO4) cause autosomal recessive anonychia. Am J Hum Genet 2006, 79(6):1105-1109. 
16. Bruchle NO, Frank J, Frank V, Senderek J, Akar A, Koc E, Rigopoulos D, van Steensel M, Zerres K, Bergmann C: RSPO4 is the major gene in autosomalrecessive anonychia and mutations cluster in the furin-like cysteine-rich domains of the Wnt signaling ligand R-spondin 4. J Invest Dermatol 2008, 128(4):791-796.

17. Nam JS, Turcotte TJ, Yoon JK: Dynamic expression of R-spondin family genes in mouse development. Gene Expr Patterns 2007, 7(3):306-312.

18. Tomaselli S, Megiorni F, Lin L, Mazzilli MC, Gerrelli D, Majore S, Grammatico P, Achermann JC: Human RSPO1/R-spondin 1 is expressed during early ovary development and augments beta-catenin signaling. PLOS One 2011, 6(1):e16366

19. Smith CA, Shoemaker CM, Roeszler KN, Queen J, Crews D, Sinclair AH: Cloning and expression of R-Spondin 1 in different vertebrates suggests a conserved role in ovarian development. BMC Dev Biol 2008, 8:72.

20. Lau Y-FC, Li Y: The human and mouse sex-determining SRY genes repress the Rspol/ $\beta$-catenin signaling. J Genet Genomics 2009, 36(4):193-202.

21. Chassot AA, Gregoire EP, Lavery R, Taketo MM, de Rooij DG, Adams IR, Chaboissier MC: RSPO1/beta-catenin signaling pathway regulates oogonia differentiation and entry into meiosis in the mouse fetal ovary. PLoS One 2011, 6(10):e25641.

22. Kondo M, Nanda I, Schmid M, Schartl M: Sex determination and sex chromosome evolution: insights from medaka. Sex Dev 2009, 3(2-3):88-98.

23. Matsuda M, Nagahama Y, Shinomiya A, Sato T, Matsuda C, Kobayashi T, Morrey CE, Shibata N, Asakawa S, Shimizu N, et al: DMY is a Y-specific DM-domain gene required for male development in the medaka fish. Nature 2002, 417(6888):559-563.

24. Nanda I, Kondo M, Hornung U, Asakawa S, Winkler C, Shimizu A, Shan Z, Haaf T, Shimizu N, Shima A, et al: A duplicated copy of DMRT1 in the sex-determining region of the $Y$ chromosome of the medaka, Oryzias latipes. Proc Natl Acad Sci USA 2002, 99(18):11778-11783.

25. Nagahama Y: Gonadal steroid hormones: major regulators of gonadal sex differentiation and gametogenesis in fish. In Proc 6th International Symposium on Reproductive Physiology of fish. Edited by Norberg B, Kjesbu OS, Taranger GL, Andersson E, Stefansson SO. Bergen: Institute of Marine Research and University of Bergen; 2000:211-222

26. Wang DS, Kobayashi T, Zhou LY, Paul-Prasanth B, ljiri S, Sakai F, Okubo K, Morohashi K, Nagahama Y: FoxI2 up-regulates aromatase gene transcription in a female-specific manner by binding to the promoter as well as interacting with ad4 binding protein/steroidogenic factor 1 . Mol Endocrinol 2007, 21(3):712-725

27. Guiguen Y, Baroiller JF, Ricordel MJ, Iseki K, McMeel OM, Martin SA, Fostier $\mathrm{A}$ : Involvement of estrogens in the process of sex differentiation in two fish species: the rainbow trout (Oncorhynchus mykiss) and a tilapia (Oreochromis niloticus). Mol Reprod Dev 1999, 54(2):154-162.

28. Nicol B, Guiguen Y: Expression profiling of Wnt signaling genes during gonadal differentiation and gametogenesis in rainbow trout. Sex Dev 2011, 5(6):318-329

29. Kobayashi T, Matsuda M, Kajiura-Kobayashi H, Suzuki A, Saito N, Nakamoto M, Shibata N, Nagahama Y: Two DM domain genes, DMY and DMRT1, involved in testicular differentiation and development in the medaka, Oryzias latipes. Developmental dynamics: an official publication of the American Association of Anatomists 2004, 231(3):518-526.

30. Shibata Y, Paul-Prasanth B, Suzuki A, Usami T, Nakamoto M, Matsuda M, Nagahama Y: Expression of gonadal soma derived factor (GSDF) is spatially and temporally correlated with early testicular differentiation in medaka. Gene Expr Patterns 2010, 10(6):283-289.

31. Kanamori A: Systematic identification of genes expressed during early oogenesis in medaka. Mol Reprod Dev 2000, 55(1):31-36.

32. Pellas TC, Ramachandran B, Duncan M, Pan SS, Marone M, Chada K: Germcell deficient $(\mathrm{gcd})$, an insertional mutation manifested as infertility in transgenic mice. Proc Natl Acad Sci USA 1991, 88(19):8787-8791.

33. Kurokawa H, Saito D, Nakamura S, Katoh-Fukui Y, Ohta K, Baba T, Morohashi K, Tanaka M: Germ cells are essential for sexual dimorphism in the medaka gonad. Proc Natl Acad Sci USA 2007, 104(43):16958-16963.

34. Slanchev K, Stebler J, de la Cueva-Mendez G, Raz E: Development without germ cells: the role of the germ line in zebrafish sex differentiation. Proc Natl Acad Sci USA 2005, 102(11):4074-4079.

35. Auguste A, Chassot AA, Grégoire EP, Renault L, Pannetier M, Treier M, Pailhoux E, Chaboissier MC: Loss of R-spondin1 and Foxl2 amplifies female-to-male sex reversal in XX mice. Sex Dev 2011, 5(6):304-317.
36. Maatouk DM, DiNapoli L, Alvers A, Parker KL, Taketo MM, Capel B: Stabilization of beta-catenin in XY gonads causes male-to-female sex-reversal. Hum Mol Genet 2008, 17(19):2949-2955.

37. Jordan BK, Mohammed M, Ching ST, Delot E, Chen XN, Dewing P, Swain A, Rao PN, Elejalde BR, Vilain E: Up-regulation of WNT-4 signaling and dosage-sensitive sex reversal in humans. Am J Hum Genet 2001, 68(5):1102-1109.

38. Thompson JD, Gibson TJ, Plewniak F, Jeanmougin F, Higgins DG: The CLUSTAL_X windows interface: flexible strategies for multiple sequence alignment aided by quality analysis tools. Nucleic Acids Res 1997, 25(24):4876-4882

39. Suzuki A, Tanaka M, Shibata N, Nagahama Y: Expression of aromatase mRNA and effects of aromatase inhibitor during ovarian development in the medaka, Oryzias latipes. J Exp Zool A Comp Exp Biol 2004, 301(3):266-273.

40. Nakamoto M, Fukasawa M, Orii S, Shimamori K, Maeda T, Suzuki A, Matsuda M, Kobayashi T, Nagahama Y, Shibata N: The cloning and expression of medaka cholesterol side chain cleavage cytochrome P450 (P450scc) during gonadal development. Dev Growth Differ 2010, 52(4):385-395.

41. Zhou LY, Wang DS, Kobayashi T, Yano A, Paul-Prasanth B, Suzuki A, Sakai F, Nagahama Y: A novel type of P450c17 lacking the lyase activity is responsible for C21-steroid biosynthesis in the fish ovary and head kidney. Endocrinology 2007, 148(9):4282-4291.

42. Hirakawa I, Miyagawa S, Katsu Y, Kagami Y, Tatarazako N, Kobayashi T, Kusano T, Mizutani T, Ogino Y, Takeuchi T, et al: Gene expression profiles in the testis associated with testis-ova in adult Japanese medaka (Oryzias latipes) exposed to 17a-ethinylestradiol. Chemosphere 2012, 87(7):668-674

43. Lange A, Paull GC, Coe TS, Katsu Y, Urushitani H, Iguchi T, Tyler CR: Sexual reprogramming and estrogenic sensitization in wild fish exposed to ethinylestradiol. Environ Sci Technol 2009, 43(4):1219-1225.

44. Kobayashi H, Iwamatsu T: Sex reversal in the medaka Oryzias latipes by brief exposure of early embryos to estradiol-17beta. Zoolog Sci 2005 22(10):1163-1167.

doi:10.1186/1471-213X-12-36

Cite this article as: Zhou et al:: R-spondins are involved in the ovarian differentiation in a teleost, medaka (Oryzias latipes). BMC Developmental Biology 2012 12:36.

\section{Submit your next manuscript to BioMed Central and take full advantage of:}

- Convenient online submission

- Thorough peer review

- No space constraints or color figure charges

- Immediate publication on acceptance

- Inclusion in PubMed, CAS, Scopus and Google Scholar

- Research which is freely available for redistribution 